\title{
Thiazolium-Mediated Multicomponent Reactions: A Facile Synthesis of 3-Aminofuran Derivatives
}

\author{
Cheng Ma* and Yewei Yang \\ Department of Chemistry, Zhejiang University, Hangzhou, China, 310027
}

General : All reactions were carried out under a nitrogen atmosphere with dry, freshly distilled solvents under anhydrous conditions. Dichloromethane was distilled from calcium hydride immediately prior to use. THF was distilled from sodium-benzophenone. All chemicals were distilled or recrystallized before use, where necessary.

All reactions were monitored by thin-layer chromatography (TLC) carried out on. Merck silica gel plates $(60 \mathrm{~F}-254)$ using UV-light $(254 \mathrm{~nm})$. Flash chromatography was performed on Merk silica gel Kieselgel 60 (230-400 mesh).

NMR spectra were recorded on AM400 or AM500 (Bruker) instruments in $\mathrm{CDCl}_{3}$. High resolution mass spectral (HRMS) analyses were measured on an APEX III (Bruker) mass spectrometer using ESI (electrospray ionosation) techniques. Infrared spectra were recorded on a Nicolet 470 FT-IR Spectrometer. Melting points are uncorrected.

\section{General Procedure:}

To a suspension of $\mathrm{NaH}(2 \mathrm{mmol})$ in anhydrous $\mathrm{CH}_{2} \mathrm{Cl}_{2}(3 \mathrm{ml})$ was added thiazolium salt 1 (1 $\mathrm{mmol})$ in dry $\mathrm{CH}_{2} \mathrm{Cl}_{2}(2 \mathrm{~mL})$ under a nitrogen atmosphere at $-78^{\circ} \mathrm{C}$. After $10 \mathrm{~min}$, a mixture of aldehyde $(0.5 \mathrm{mmol})$ and DMAD $(0.75 \mathrm{mmol})$ in $\mathrm{CH}_{2} \mathrm{Cl}_{2}(2 \mathrm{ml})$ was added over $10 \mathrm{~min}$, then stirred at this temperature for 2 hours. The reaction temperature was then raised slowly to $0^{\circ} \mathrm{C}$ in $1 \mathrm{~h}$, and for additional 2 hours at $0^{\circ} \mathrm{C}$. On completion of the reaction, the reaction mixture was carefully poured into a solution of $\mathrm{NaHCO}_{3}$ (sat. solution, $50 \mathrm{ml}$ ), then extracted with $\mathrm{CH}_{2} \mathrm{Cl}_{2}(50 \mathrm{ml})$ twice. The organics phase was washed with brine and dried with anhydrous $\mathrm{Na}_{2} \mathrm{SO}_{4}$. The solvent was removed under reduced pressure and the resulting oil was purified by column chromatography using appropriate hexane-ethyl acetate solvent mixture to afford the products (3a-m).

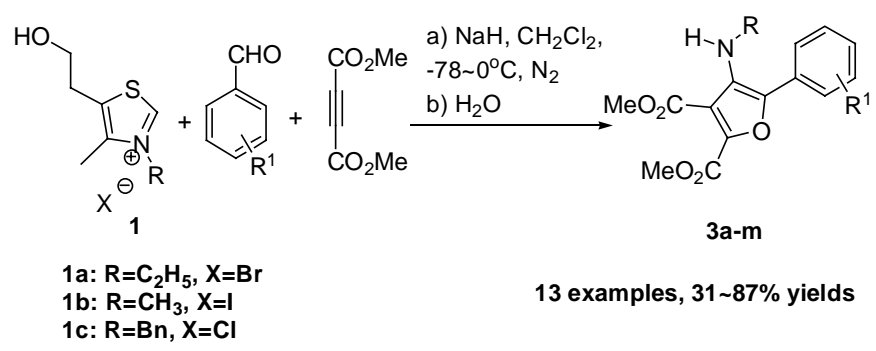


3a: Pale brown crystalline solid; m.p. $134-135^{\circ} \mathrm{C}$; IR (KBr) $v_{\max }: 3328,2955,1724,1709,1595,1508$, 1445, 1335, $1211 \mathrm{~cm}^{-1} ;{ }^{1} \mathbf{H}$ NMR (500 MHz, $\left.\mathrm{CDCl}_{3}\right) \delta 8.28(\mathrm{~d}, J=9.0,2 \mathrm{H}), 7.95(\mathrm{~d}, J=9.0,2 \mathrm{H}), 3.96$ (s, 6H), 2.97 (q, $J=7.1,2 \mathrm{H}), 1.15$ (t, $J=7.1,3 \mathrm{H}) ;{ }^{13} \mathbf{C}$ NMR $\left(100 \mathrm{MHz}, \mathrm{CDCl}_{3}\right) \delta 163.5,158.2,146.4$, 142.7, 139.8, 136.6, 135.7, 125.6, 124.0, 119.0, 52.7, 52.5, 43.3, 15.7 .

HRMS (ESI): calculated for $\mathrm{C}_{16} \mathrm{H}_{17} \mathrm{~N}_{2} \mathrm{O}_{7} \mathrm{Na}[\mathrm{M}+\mathrm{Na}]^{+} 371.0850$, found 371.0855 .

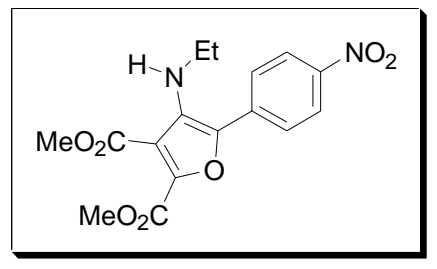

3b: Pale yellow solid; m.p. $109-110^{\circ} \mathrm{C}$; IR (KBr) $v_{\max }$ : 3345, 2960, 2869, 1730, 1701, 1608, 1528, 1439, 1352, 1232, $1209 \mathrm{~cm}^{-1} ;{ }^{1} \mathbf{H}$ NMR $\left(500 \mathrm{MHz}, \mathrm{CDCl}_{3}\right) \delta 8.68(\mathrm{~s}, 1 \mathrm{H}), 8.13(\mathrm{~d}, J=7.6,2 \mathrm{H}), 7.61$ (t, $J=8.0,1 \mathrm{H}), 3.96(\mathrm{~s}, 6 \mathrm{H}), 2.95$ (q, $J=7.0,2 \mathrm{H}), 1.16(\mathrm{t}, J=7.1,3 \mathrm{H}) ;{ }^{13} \mathrm{C}$ NMR $\left(125 \mathrm{MHz}, \mathrm{CDCl}_{3}\right) \delta$ 163.7, 158.4, 148.8, 142.2, 140.1, 135.4, 131.5, 131.0, 129.8, 122.4, 120.4, 119.4, 52.8, 52.7, 43.4, 15.8 .

HRMS (ESI): calculated for $\mathrm{C}_{16} \mathrm{H}_{16} \mathrm{~N}_{2} \mathrm{O}_{7} \mathrm{Na}[\mathrm{M}+\mathrm{Na}]^{+}$371.0850, found 371.0856.

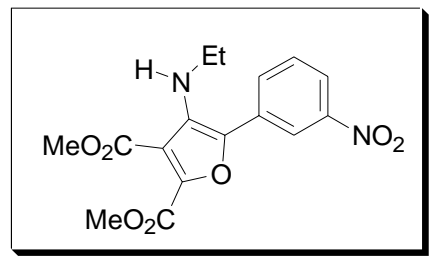

3c: Pale yellow viscous oil; IR (film) $v_{\max }$ : 3384, 2952, 2870, 1735, 1620, 1541, 1438, 1331, 1209, $1140 \mathrm{~cm}^{-1} ;{ }^{1} \mathbf{H}$ NMR $\left(500 \mathrm{MHz}, \mathrm{CDCl}_{3}\right) \delta$ 7.52-7.31 (m, 4H), 4.25 (br s, 1H), 3.94 (s, 3H), 3.91 (s, $3 \mathrm{H}), 2.75$ (q, $J=7.1,2 \mathrm{H}), 1.02$ (t, $J=7.1,3 \mathrm{H}) ;{ }^{13} \mathbf{C}$ NMR $\left(125 \mathrm{MHz}, \mathrm{CDCl}_{3}\right) \delta 164.3,158.5,142.4$, $135.7,135.2,135.0,132.6,130.7,130.0,129.9,126.5,115.6,52.6,52.4,40.8,15.5$.

HRMS (ESI): calculated for $\mathrm{C}_{16} \mathrm{H}_{16} \mathrm{NO}_{5} \mathrm{ClNa}[\mathrm{M}+\mathrm{Na}]^{+}$360.0609, found 360.0601 .

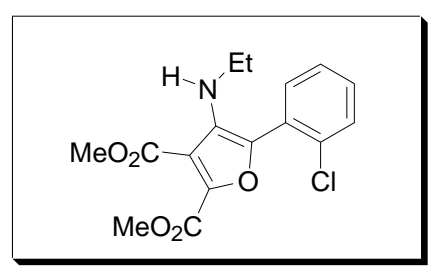

3d: Pale yellow solid; m.p. $64-66^{\circ} \mathrm{C}$; IR (KBr) $v_{\max }$ : 3357, 2955, 2871, 1730, 1701, 1611, 1538, 1441, 1335, 1232, $1209 \mathrm{~cm}^{-1} ;{ }^{1} \mathbf{H}$ NMR $\left(500 \mathrm{MHz}, \mathrm{CDCl}_{3}\right) \delta$ 7.78-7.36 (m, 4H), 3.94-3.91 (m, 6H), 2.90-2.73 (m, 2H), 1.12-1.07 (m, 3H); ${ }^{13} \mathbf{C}$ NMR $\left(100 \mathrm{MHz}, \mathrm{CDCl}_{3}\right) \delta 163.9,158.4,141.8,141.0$, 
$133.8,133.7,129.7,128.8,128.7,126.9,119.6,52.4,42.9,15.6$.

HRMS (ESI): calculated for $\mathrm{C}_{16} \mathrm{H}_{16} \mathrm{NO}_{5} \mathrm{CINa}[\mathrm{M}+\mathrm{Na}]^{+} 360.0609$, found 360.0613 .

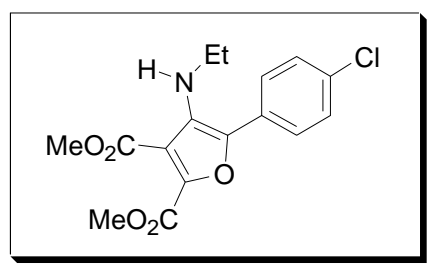

3e: Pale yellow viscous oil; IR (film) $v_{\max }$ : 3354, 2954, 2865, 1731, 1604, 1503, 1438, 1331, 1223 , $1206 \mathrm{~cm}^{-1} ;{ }^{1} \mathbf{H}$ NMR $\left(400 \mathrm{MHz}, \mathrm{CDCl}_{3}\right) \delta 7.82$ (q, $\left.J=5.6,2 \mathrm{H}\right), 7.12$ (t, $\left.J=8.4,2 \mathrm{H}\right), 3.70$ (br s, $\left.1 \mathrm{H}\right)$, $3.95(\mathrm{~s}, 3 \mathrm{H}), 3.93(\mathrm{~s}, 3 \mathrm{H}), 2.92(\mathrm{q}, J=6.8,2 \mathrm{H}), 1.10(\mathrm{t}, J=6.8,3 \mathrm{H}) ;{ }^{13} \mathbf{C}$ NMR $\left(100 \mathrm{MHz}, \mathrm{CDCl}_{3}\right) \delta$ $163.9,163.6,161.2,158.4,142.2,140.7,133.0,127.8,127.7,126.2,119.7,115.8,115.6,52.4,52.3$, $42.8,15.6$.

HRMS (ESI): calculated for $\mathrm{C}_{16} \mathrm{H}_{16} \mathrm{NO}_{5} \mathrm{FNa}[\mathrm{M}+\mathrm{Na}]^{+} 344.0905$, found 344.0911 .

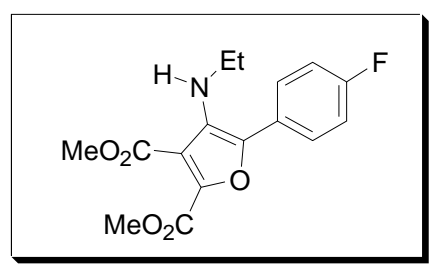

3f: Pale yellow viscous oil; IR (film) $v_{\max }: 3378,2955,2870,1732,1614,1547,1477,1337,1239$, 1210, $1119 \mathrm{~cm}^{-1} ;{ }^{1} \mathbf{H}$ NMR (400 MHz, $\left.\mathrm{CDCl}_{3}\right) \delta$ 7.35-7.12 (m, 3H), 4.20 (br s, $\left.1 \mathrm{H}\right), 3.93-3.91(\mathrm{~m}, 6 \mathrm{H})$, 2.90-2.84 (m, 2H), 1.09-1.06 (m, 3H); ${ }^{13} \mathbf{C}$ NMR $\left(100 \mathrm{MHz}, \mathrm{CDCl}_{3}\right) \delta 164.0,158.3,142.8,135.6$, $132.5,125.3,125.2,124.1,120.9,120.8,117.5,117.3,116.6,52.5,52.4,41.2,15.4$.

HRMS (ESI): calculated for $\mathrm{C}_{16} \mathrm{H}_{15} \mathrm{NO}_{5} \mathrm{~F}_{2} \mathrm{Na}[\mathrm{M}+\mathrm{Na}]^{+}$362.0811, found 362.0816 .

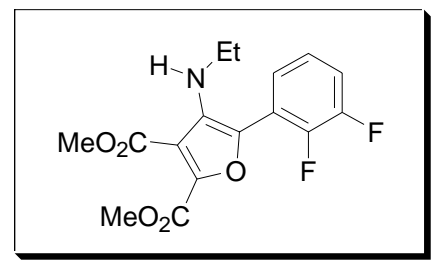

3g: Pale yellow solid; m.p. $67-68^{\circ} \mathrm{C}$; IR (KBr) $v_{\max }$ : 3379, 2948, 1728, 1702, 1608, 1443, 1232, 1207 $\mathrm{cm}^{-1} ;{ }^{1} \mathbf{H}$ NMR $\left(500 \mathrm{MHz}, \mathrm{CDCl}_{3}\right) \delta$ 7.77-7.31 (m, 5H), 3.94 (s, 3H), $3.92(\mathrm{~s}, 3 \mathrm{H}), 2.69(\mathrm{~s}, 3 \mathrm{H}) ;{ }^{13} \mathbf{C}$ NMR $\left(125 \mathrm{MHz}, \mathrm{CDCl}_{3}\right) \delta 164.2,158.6,142.0,140.9,134.9,130.0,128.7,128.2,126.3,118.7,52.6$, $52.5,34.9$.

HRMS (ESI): calculated for $\mathrm{C}_{15} \mathrm{H}_{15} \mathrm{NO}_{5} \mathrm{Na}[\mathrm{M}+\mathrm{Na}]^{+} 312.0842$, found 312.0842 . 


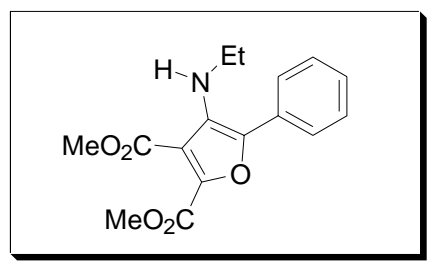

3h: Pale yellow viscous oil; IR (film) $v_{\max }$ : 3355, 2952, 2863, 1728, 1608, 1504, 1438, 1330, 1227, $1205 \mathrm{~cm}^{-1} ;{ }^{1} \mathbf{H}$ NMR $\left(500 \mathrm{MHz}, \mathrm{CDCl}_{3}\right) \delta 7.71(\mathrm{~d}, J=8.1,2 \mathrm{H}), 7.23(\mathrm{~d}, J=8.0,2 \mathrm{H}), 4.20(\mathrm{br} \mathrm{s}, 1 \mathrm{H})$, 3.93 (s, 3H), 3.91 (s, 3H), 2.94 (q, $J=7.1,2 \mathrm{H}), 2.37$ (s, 3H), 1.09 (t, $J=7.1,3 \mathrm{H}) ;{ }^{13}$ C NMR (125MHz, $\left.\mathrm{CDCl}_{3}\right) \delta 164.2,158.6,143.3,140.4,138.3,132.9,129.4,127.3,125.9,119.9,52.5,52.4,42.9,21.5$, 15.8 .

HRMS (ESI): calculated for $\mathrm{C}_{17} \mathrm{H}_{19} \mathrm{NO}_{5} \mathrm{Na}[\mathrm{M}+\mathrm{Na}]^{+} 340.1155$, found 340.1158 .

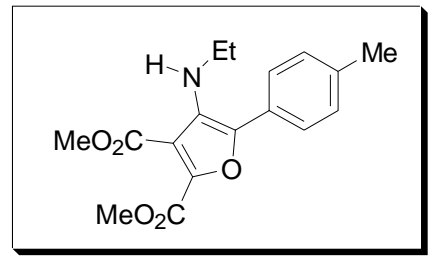

3i: Pale yellow viscous oil; IR (film) $v_{\max }$ : 3354, 2953, 2839, 1727, 1606, 1504, 1439, 1331, 1251 $\mathrm{cm}^{-1} ;{ }^{1} \mathbf{H}$ NMR $\left(500 \mathrm{MHz}, \mathrm{CDCl}_{3}\right) \delta 7.77$ (d, $\left.J=8.8,2 \mathrm{H}\right), 6.95$ (d, $\left.J=8.8,2 \mathrm{H}\right), 3.94$ (s, 3H), 3.91 (s, $3 \mathrm{H}), 3.84$ (s, 3H), 2.93 (q, $J=7.1,2 \mathrm{H}), 1.09$ (t, $J=7.1,3 \mathrm{H}) ;{ }^{13} \mathbf{C} \mathbf{N M R}\left(125 \mathrm{MHz}, \mathrm{CDCl}_{3}\right) \delta 164.2,159.7$, 158.6, 143.6, 140.0, 132.1, 127.5, 122.7, 120.2, 114.2, 55.4, 52.5, 52.4, 42.9, 15.7 .

HRMS (ESI): calculated for $\mathrm{C}_{16} \mathrm{H}_{19} \mathrm{NO}_{6} \mathrm{Na}[\mathrm{M}+\mathrm{Na}]^{+}$356.1105, found 356.1105 .

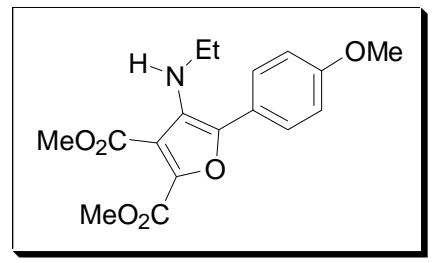

3j: Pale yellow crystalline solid; m.p. $76^{\circ} \mathrm{C}$; IR (KBr) $v_{\max }$ : 3371, 2954, 1725, 1704, 1590, 1498, 1443, 1334, 1233, $1204 \mathrm{~cm}^{-1} ;{ }^{1} \mathbf{H}$ NMR $\left(500 \mathrm{MHz}, \mathrm{CDCl}_{3}\right) \delta$ 7.76-7.74 (m, 2H), 7.11 (t, J=8.5, 2H), 3.93 (s, 3H), 3.91 (s, 3H), 2.66 (s, 3H); ${ }^{13}$ C NMR $\left(125 \mathrm{MHz}, \mathrm{CDCl}_{3}\right) \delta 164.0,163.5,161.5,158.5,141.4$, $140.9,134.6,128.2,128.1,126.3,126.2,52.5,52.4,34.9$.

HRMS (ESI): calculated for $\mathrm{C}_{15} \mathrm{H}_{15} \mathrm{FNO}_{5}[\mathrm{M}+\mathrm{H}]^{+}$308.0929, found 308.0924 .

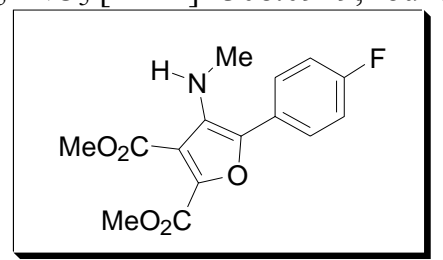


3k: Pale yellow solid; m.p. 67-68 ${ }^{\circ}$; IR (KBr) $v_{\max }$ : 3379, 2948, 1728, 1702, 1608, 1443, 1232, 1207 $\mathrm{cm}^{-1} ;{ }^{1} \mathbf{H}$ NMR $\left(500 \mathrm{MHz}, \mathrm{CDCl}_{3}\right) \delta$ 7.77-7.31 (m, 5H), $3.94(\mathrm{~s}, 3 \mathrm{H}), 3.92(\mathrm{~s}, 3 \mathrm{H}), 2.69(\mathrm{~s}, 3 \mathrm{H}) ;{ }^{13} \mathbf{C}$ NMR $\left(125 \mathrm{MHz}, \mathrm{CDCl}_{3}\right) \delta 164.2,158.6,142.0,140.9,134.9,130.0,128.7,128.2,126.3,118.7,52.6$, 52.5, 34.9. HRMS (ESI): calculated for $\mathrm{C}_{15} \mathrm{H}_{15} \mathrm{NO}_{5} \mathrm{Na}[\mathrm{M}+\mathrm{Na}]^{+} 312.0842$, found 312.0842 .

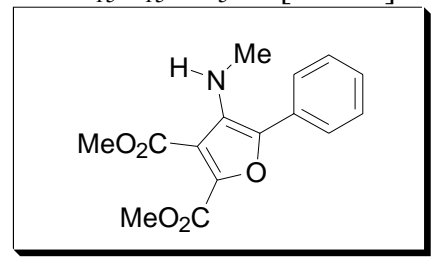

31: Pale yellow solid; m.p. $81-82^{\circ} \mathrm{C}$; IR (KBr) $v_{\max }$ : 3369, 2951, 1726, 1703, 1608, 1509, 1445, 1335, 1236,1211 cm ${ }^{-1} ;{ }^{1} \mathbf{H}$ NMR $\left(500 \mathrm{MHz}, \mathrm{CDCl}_{3}\right) \delta 7.66(\mathrm{~d}, J=8.1,2 \mathrm{H}), 7.22(\mathrm{~d}, J=7.9,2 \mathrm{H}), 3.93(\mathrm{~s}, 3 \mathrm{H})$, 3.91 (s, 3H), 2.68 (s, 3H), 2.37 (s, 3H); ${ }^{13}$ C NMR (125MHz, CDCl3) $\delta 164.1,158.5,142.5,140.5$, $138.1,134.3,129.3,127.2,126.2,118.9,52.4,52.3,34.8,21.5$.

HRMS (ESI): calculated for $\mathrm{C}_{16} \mathrm{H}_{18} \mathrm{NO}_{5}[\mathrm{M}+\mathrm{H}]^{+} 304.1179$, found 304.1176 .

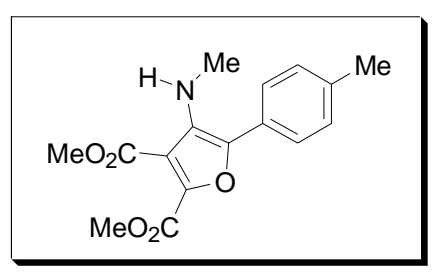

3m: Pale yellow crystalline solid; m.p. $90-90.5^{\circ} \mathrm{C}$; IR (KBr) $v_{\max }$ : 3369, 2954, 1734, 1729, 1606, 1500 , 1437, 1327, 1221, $1202 \mathrm{~cm}^{-1} ;{ }^{1} \mathbf{H}$ NMR (500 MHz, $\left.\mathrm{CDCl}_{3}\right) \delta$ 7.89-7.86 (m, 2H), 7.28-7.25 (m, 3H), 7.15-7.14 (m, 4H), 4.50 (br s, 1H), 4.03 (s, 2H), 3.91 (s, 3H), 3.82 (s, 3H); ${ }^{13}$ C NMR $(125 \mathrm{MHz}$, $\left.\mathrm{CDCl}_{3}\right) \delta 163.8,163.6,161.7,158.5,142.9,140.8,139.1,132.5,128.8,128.1,128.0,127.6,126.2$, $126.1,120.2,116.1,115.9,52.6,52.5,52.3$.

HRMS (ESI): calculated for $\mathrm{C}_{21} \mathrm{H}_{18} \mathrm{FNO}_{5} \mathrm{Na}[\mathrm{M}+\mathrm{Na}]^{+} 406.1061$, found 406.1060 .

\begin{tabular}{|l|l|}
\hline & \\
\hline$M_{2} \mathrm{O}_{2} \mathrm{C}$ & $\mathrm{O}$ \\
\hline $\mathrm{MeO}_{2} \mathrm{C}$ & $\mathrm{Bn}$ \\
\hline
\end{tabular}




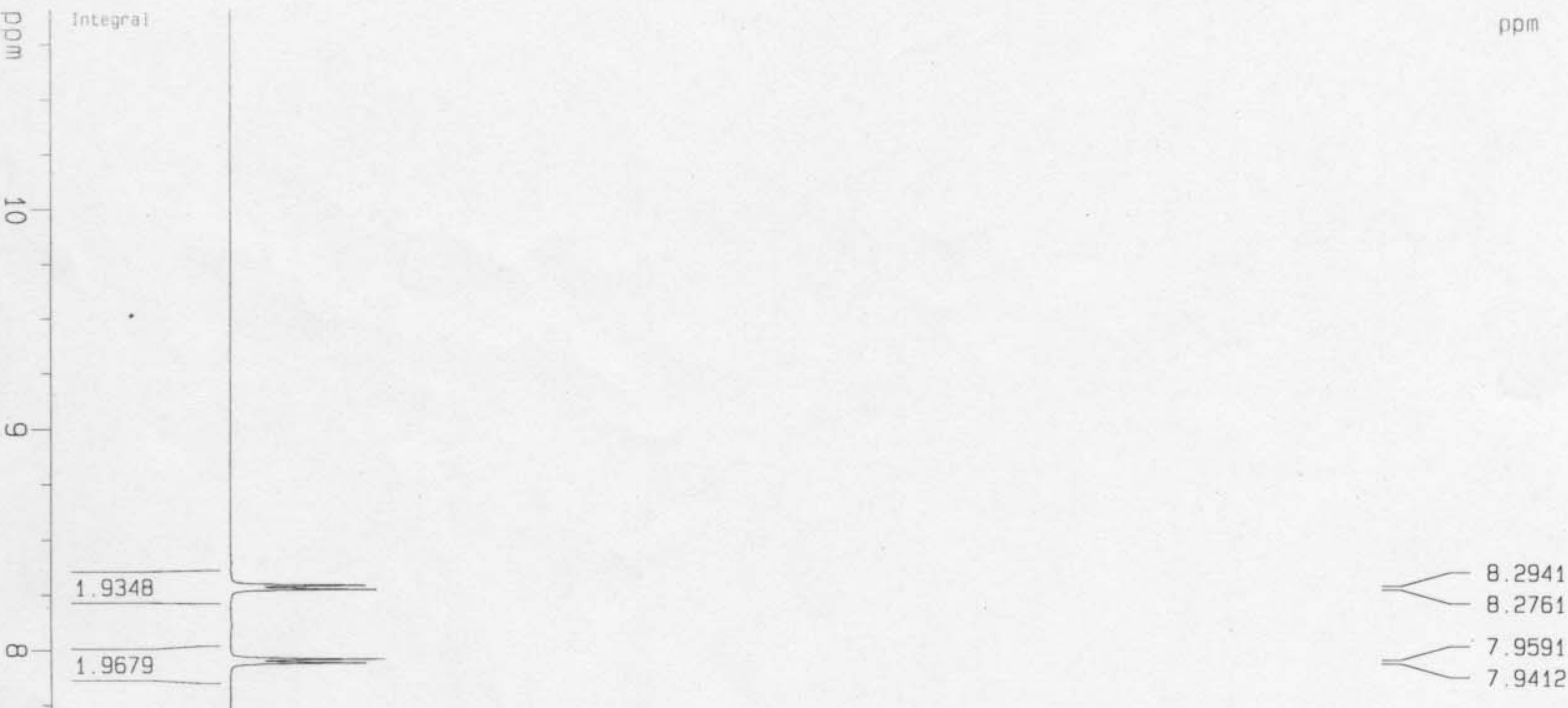

7.2680

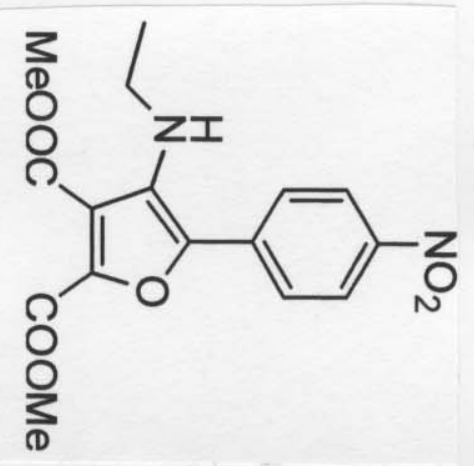

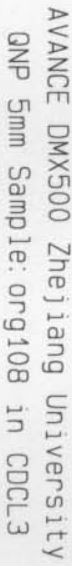

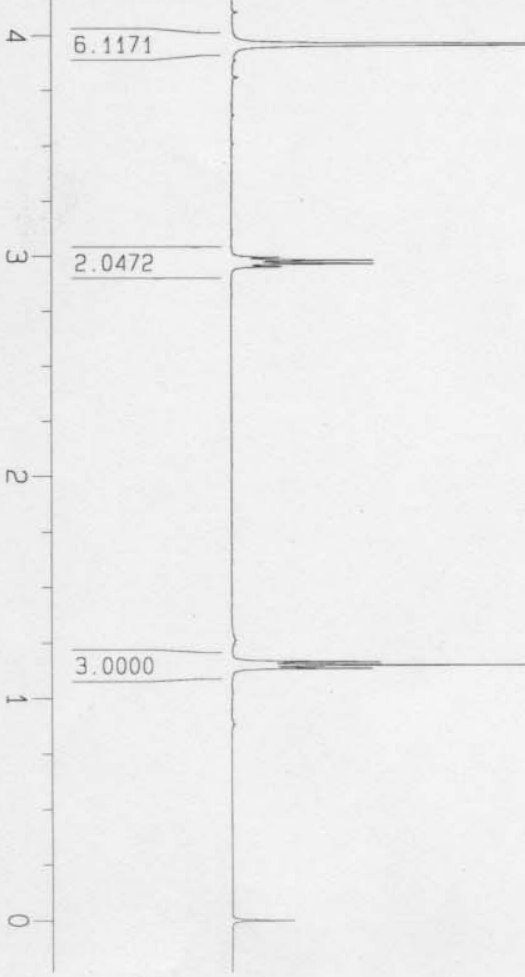

3.9576

2. 9928 

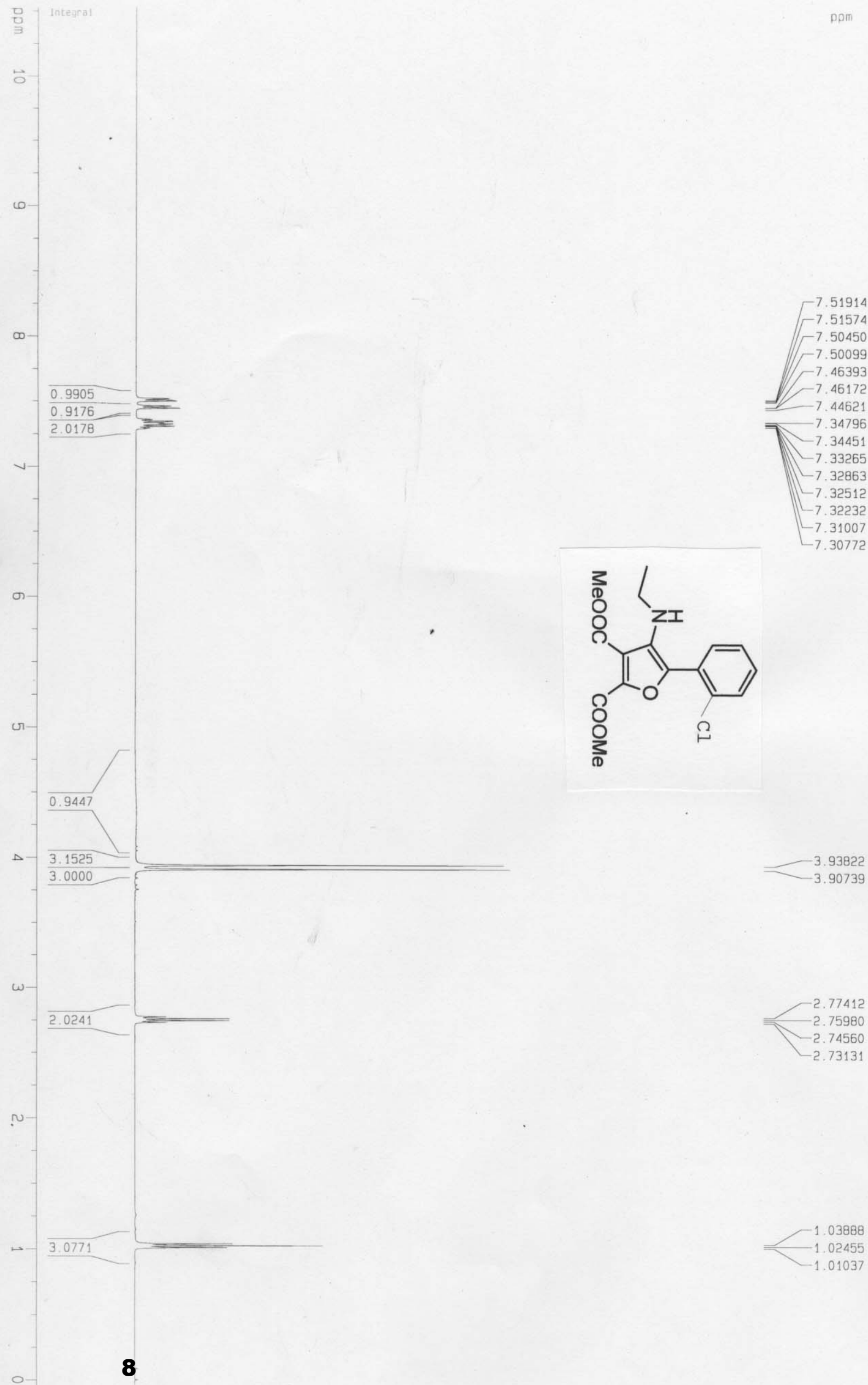


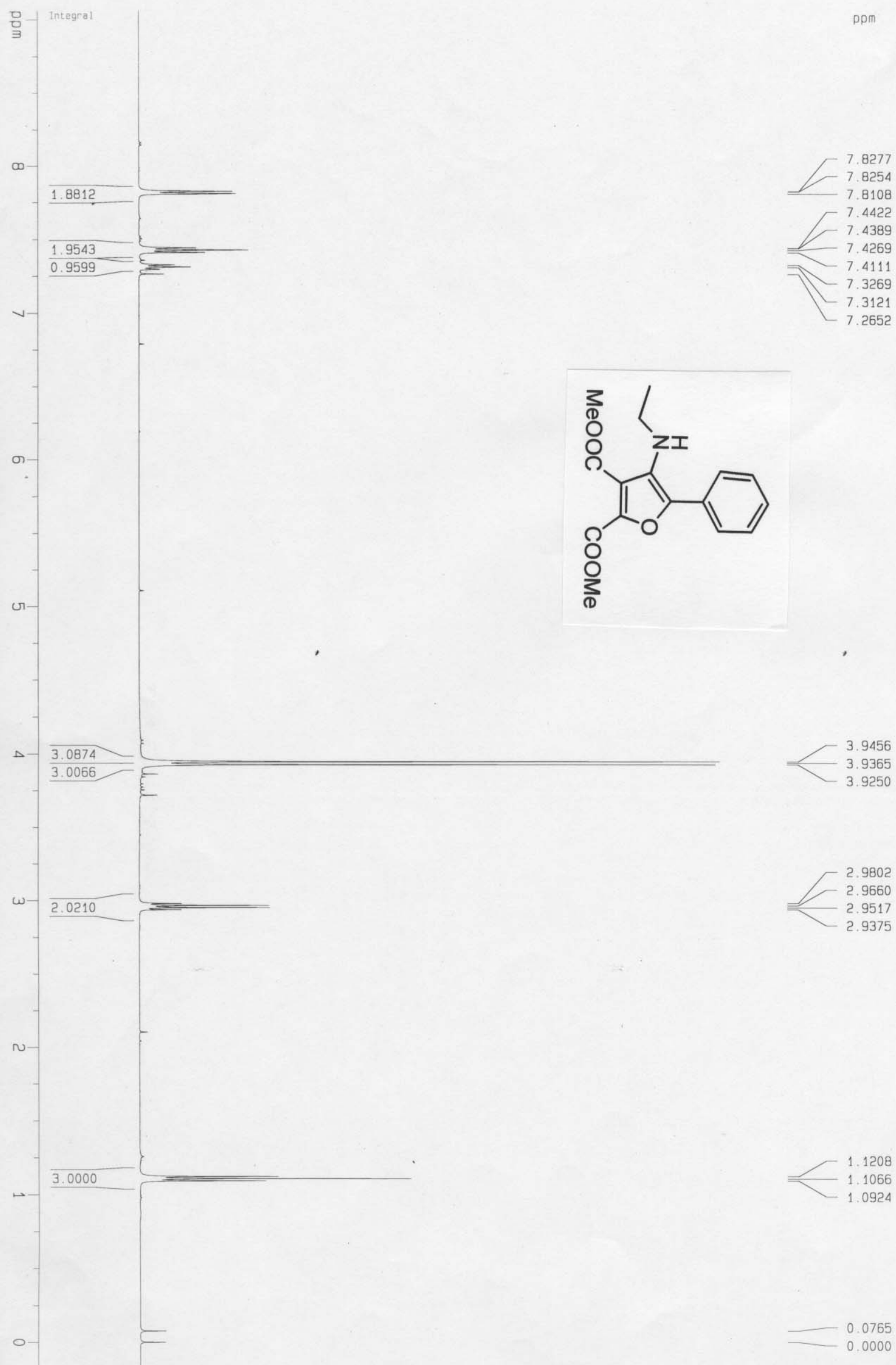


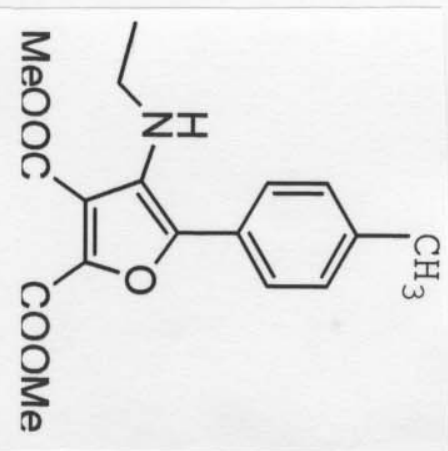

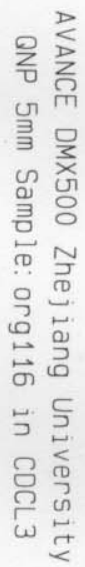

$\triangle-$

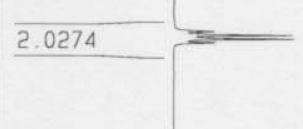


$\infty$

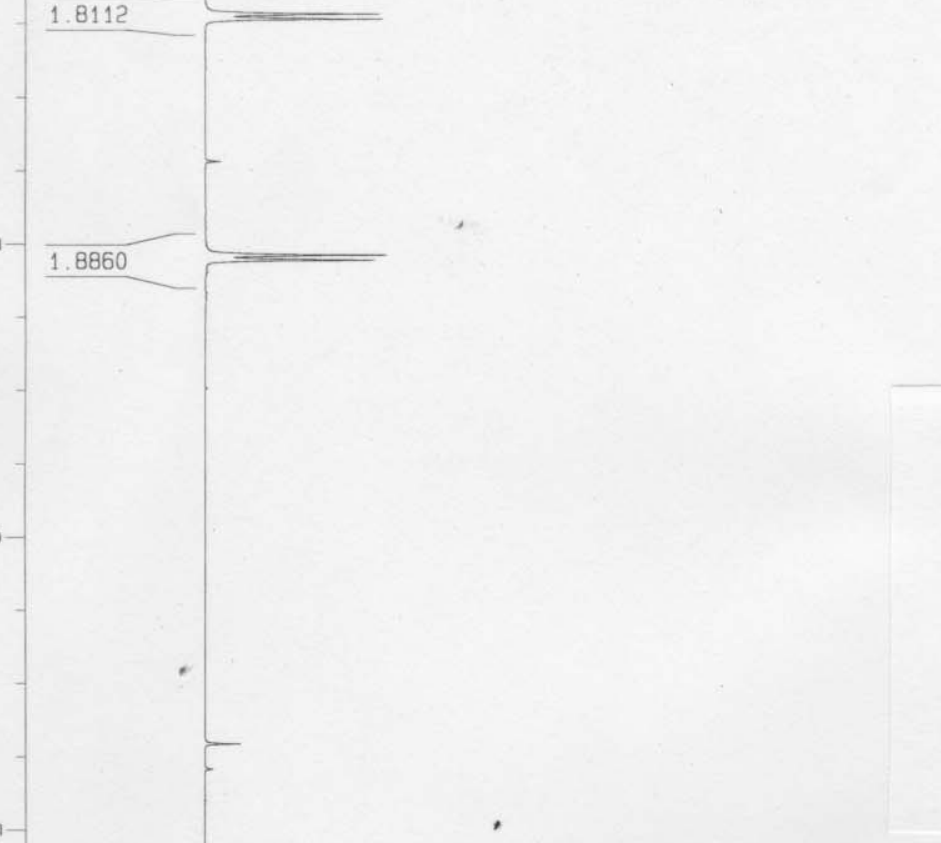

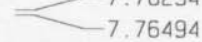

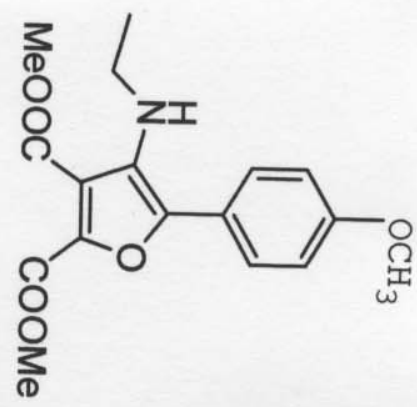

닌

जा

当은

ल)

옴

는

$\therefore$

은

음

ㄴ. 돈

ก

อำ 

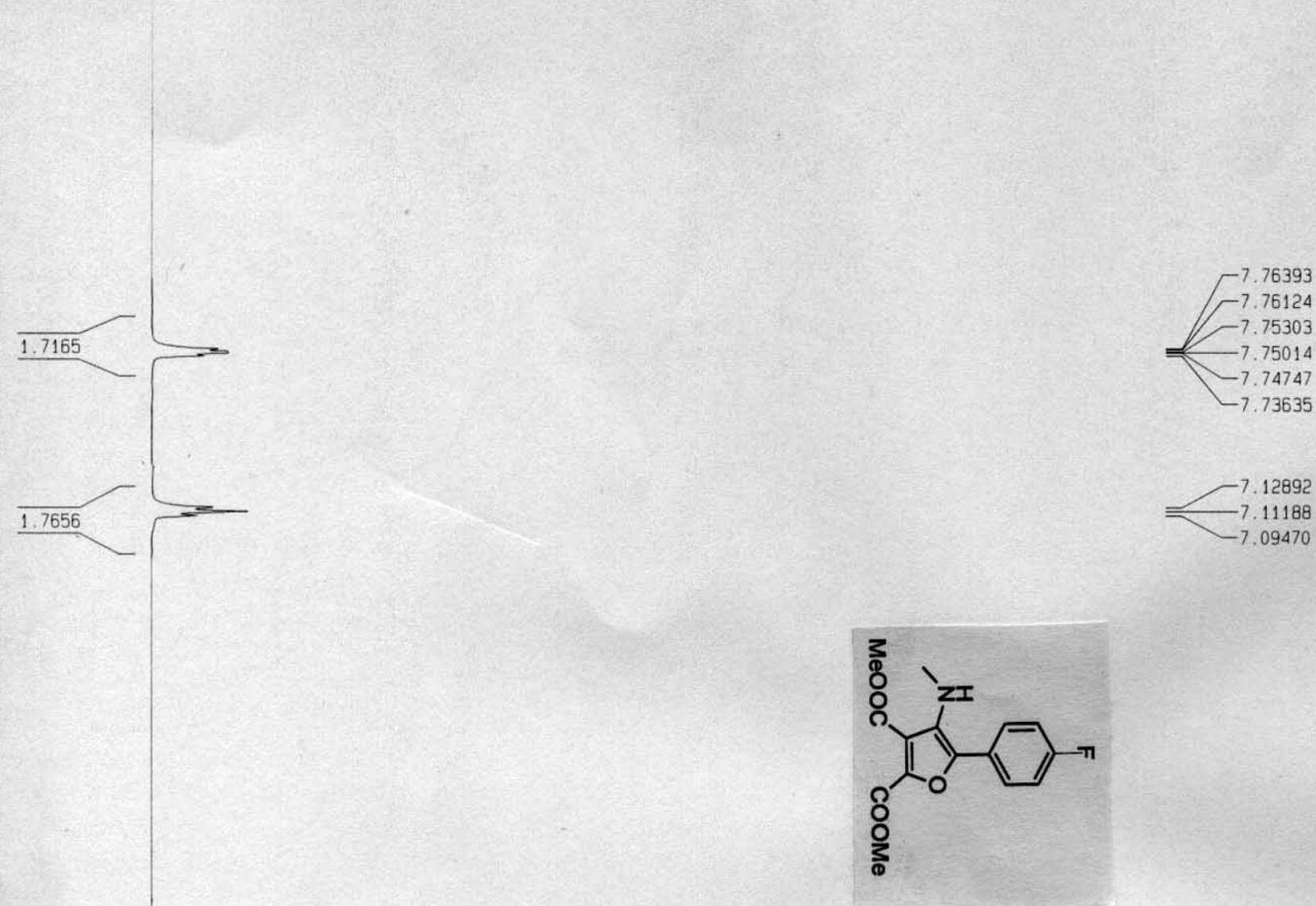

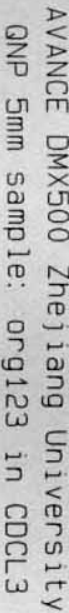
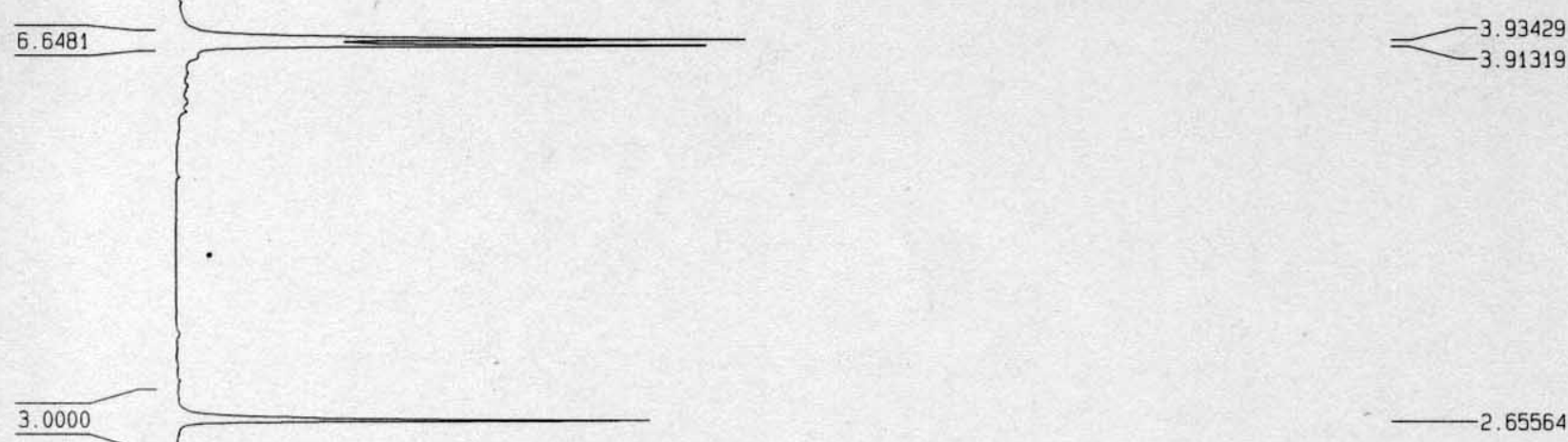

2. 65564 


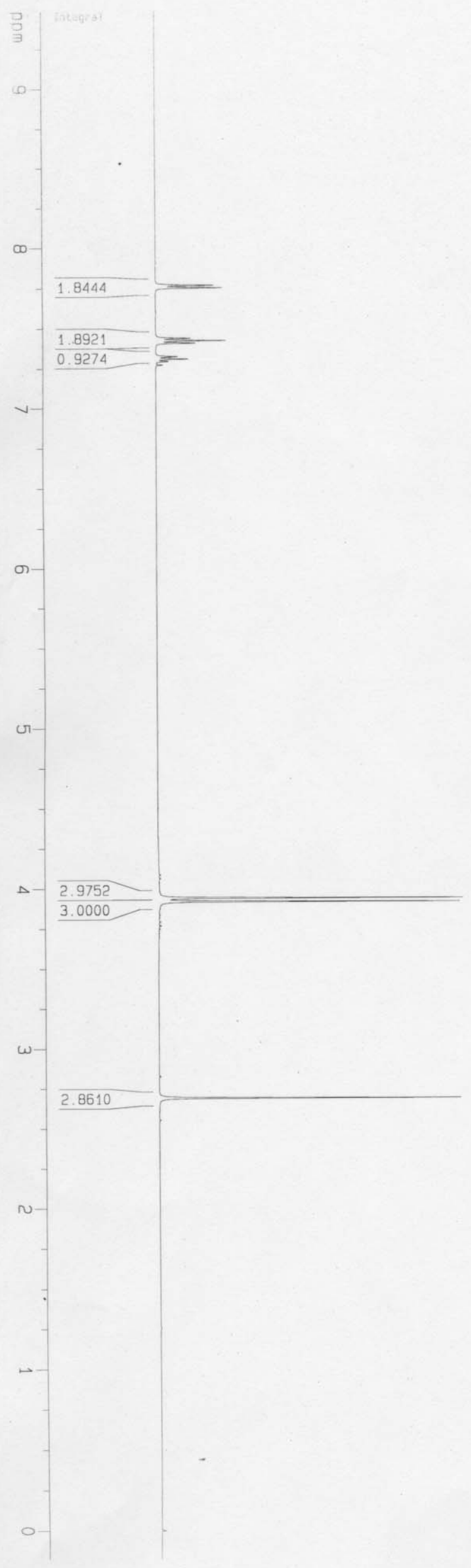

$\rho p m$

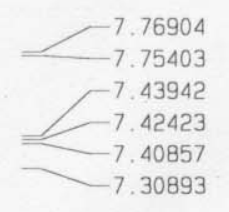<smiles>[CH][C+](C)c1c(-c2ccccc2)oc([O-])c1[O-]</smiles>

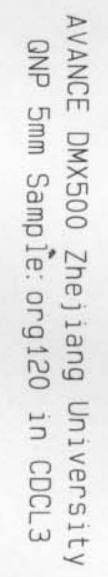

16 


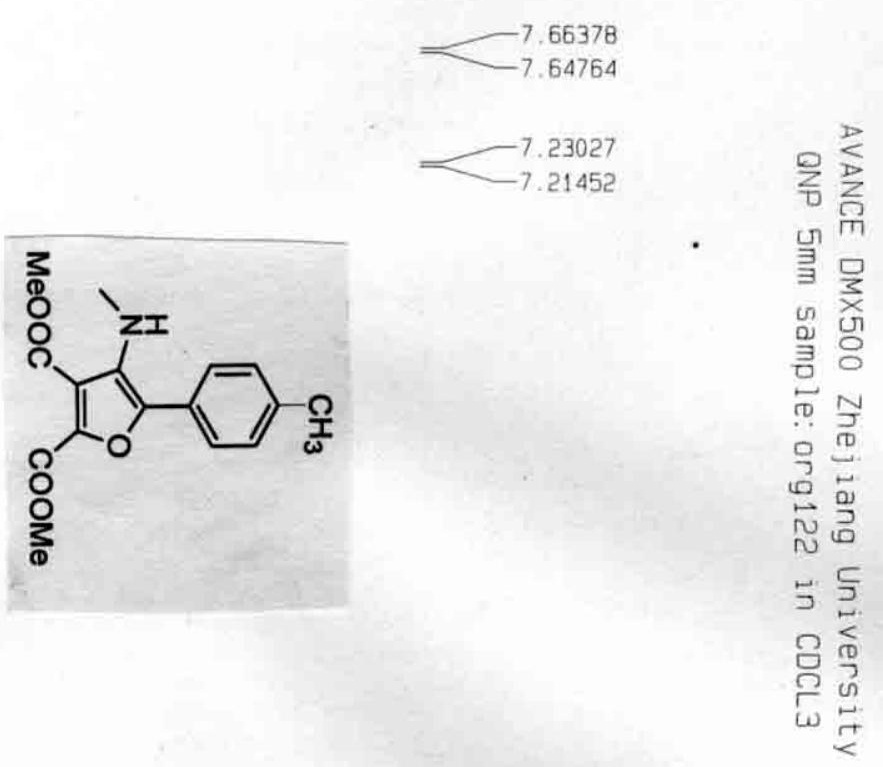

$\quad 3.93209$
$=-3.90672$

2.6744

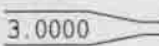




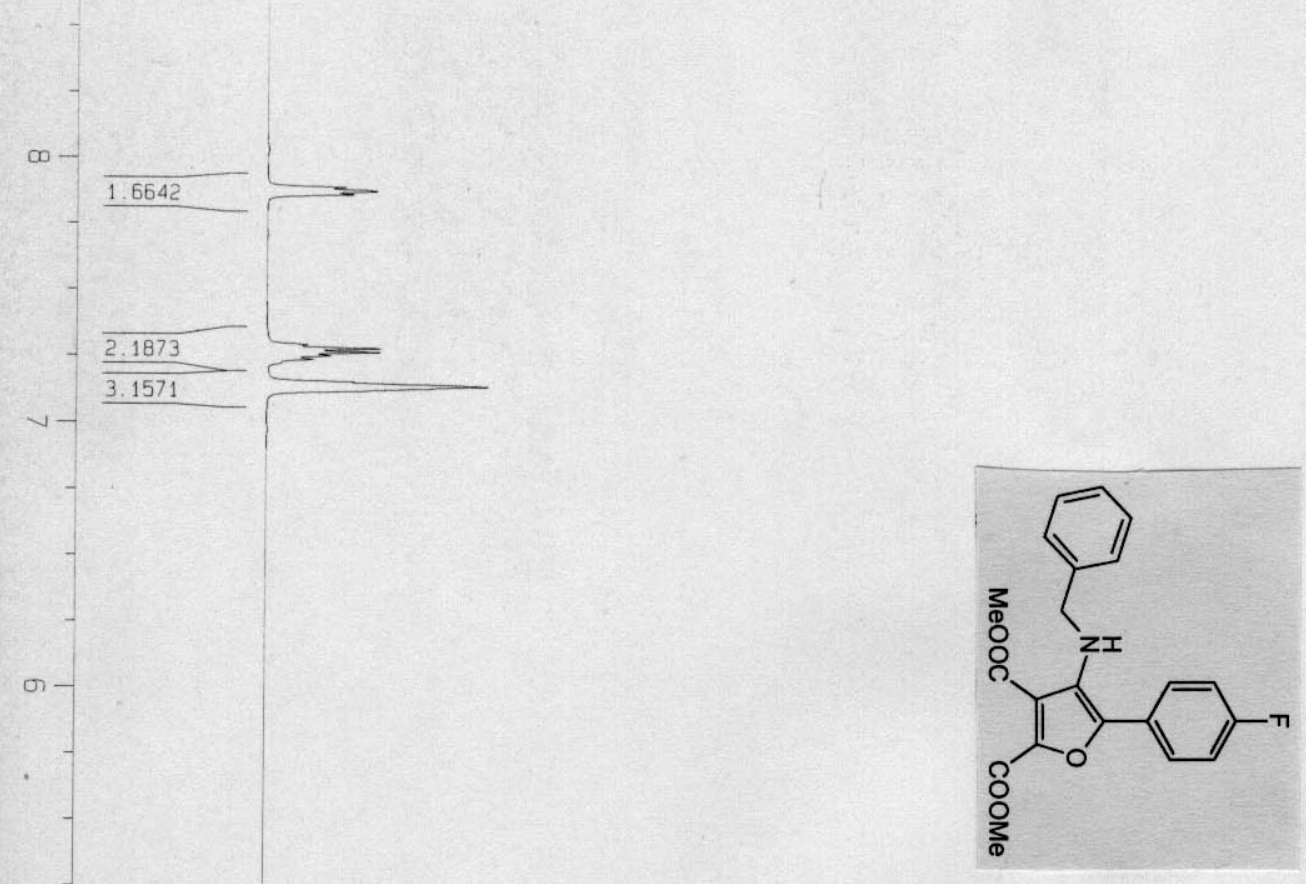

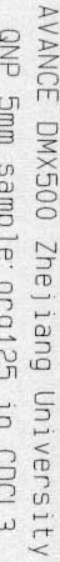
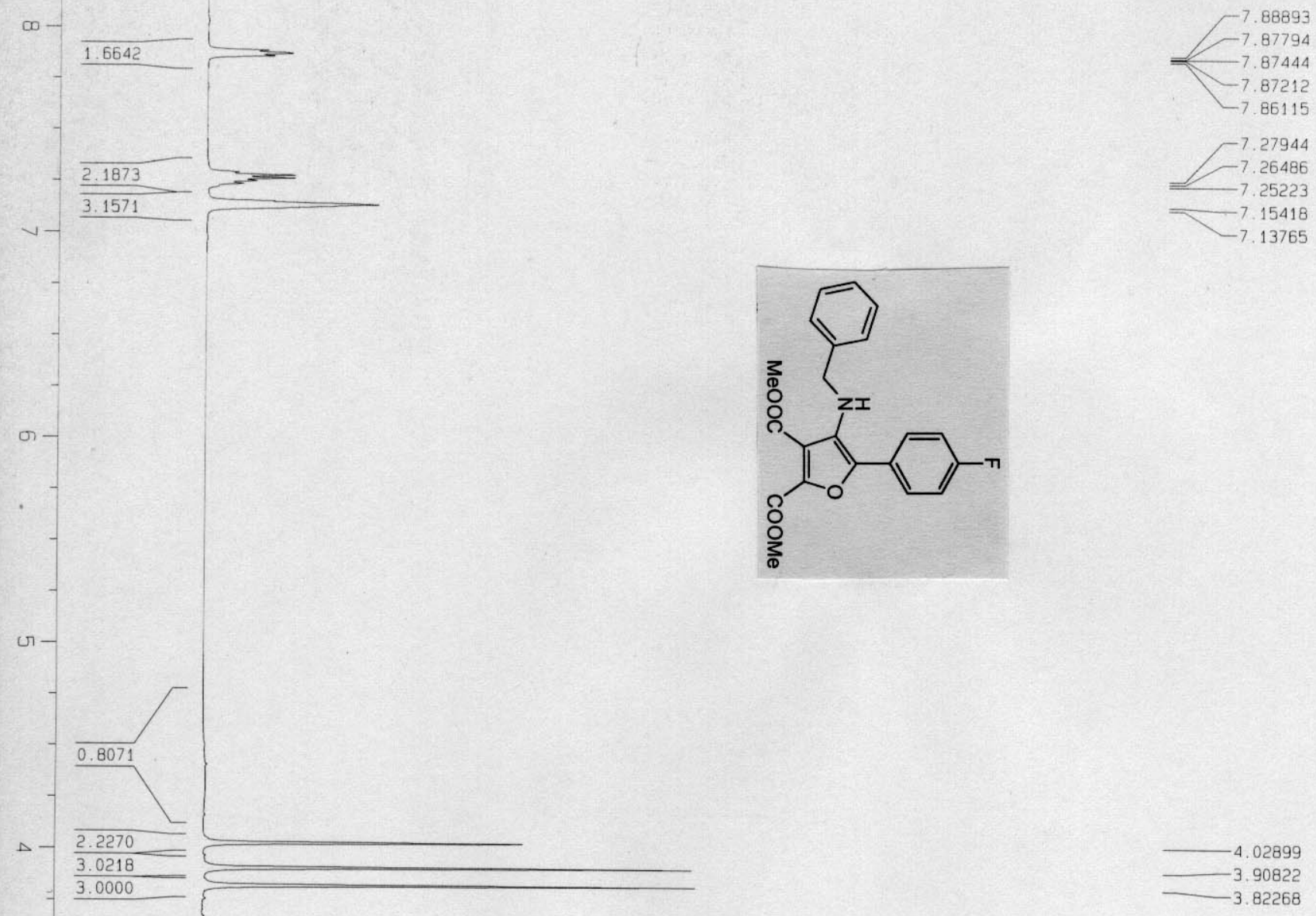


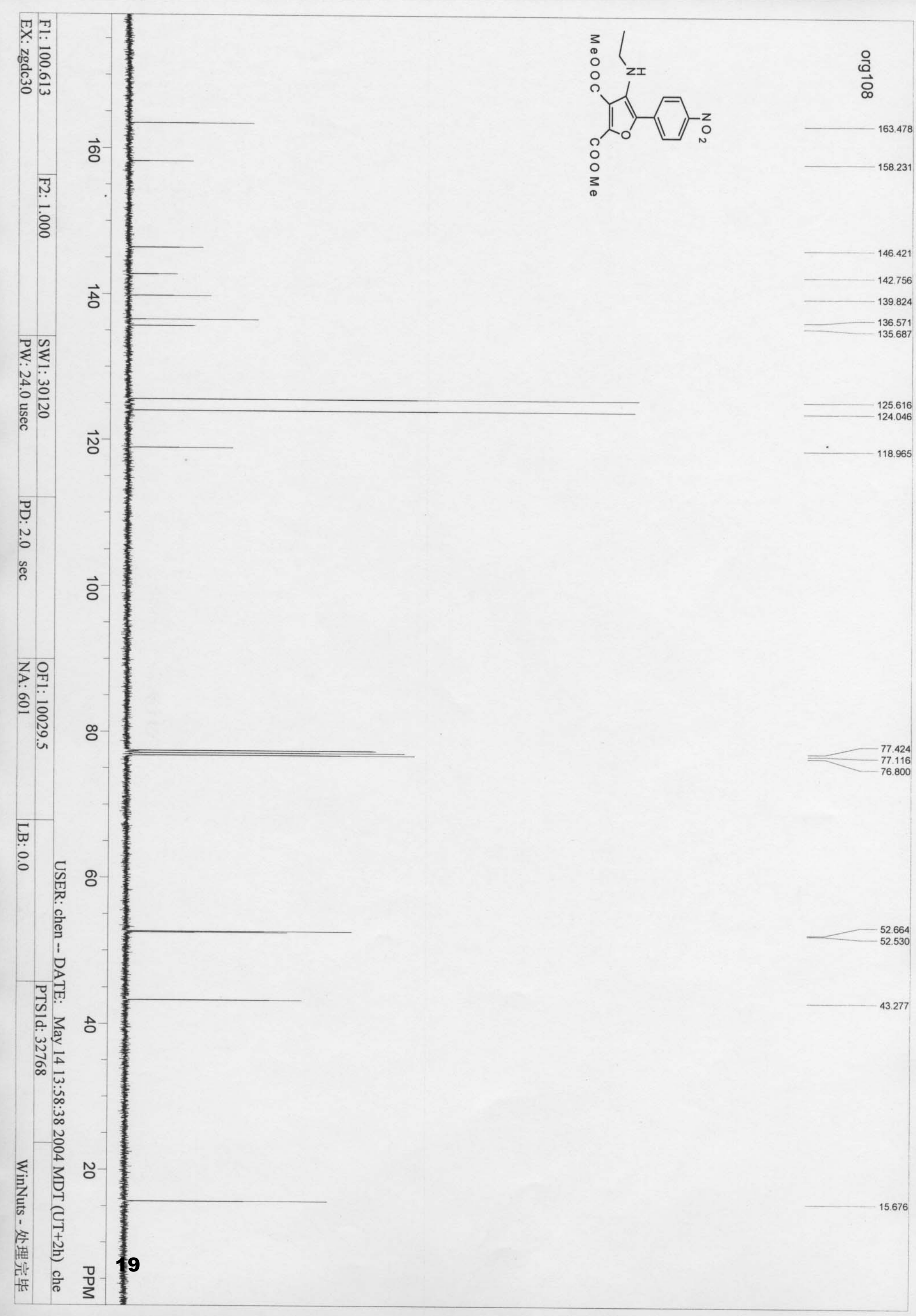




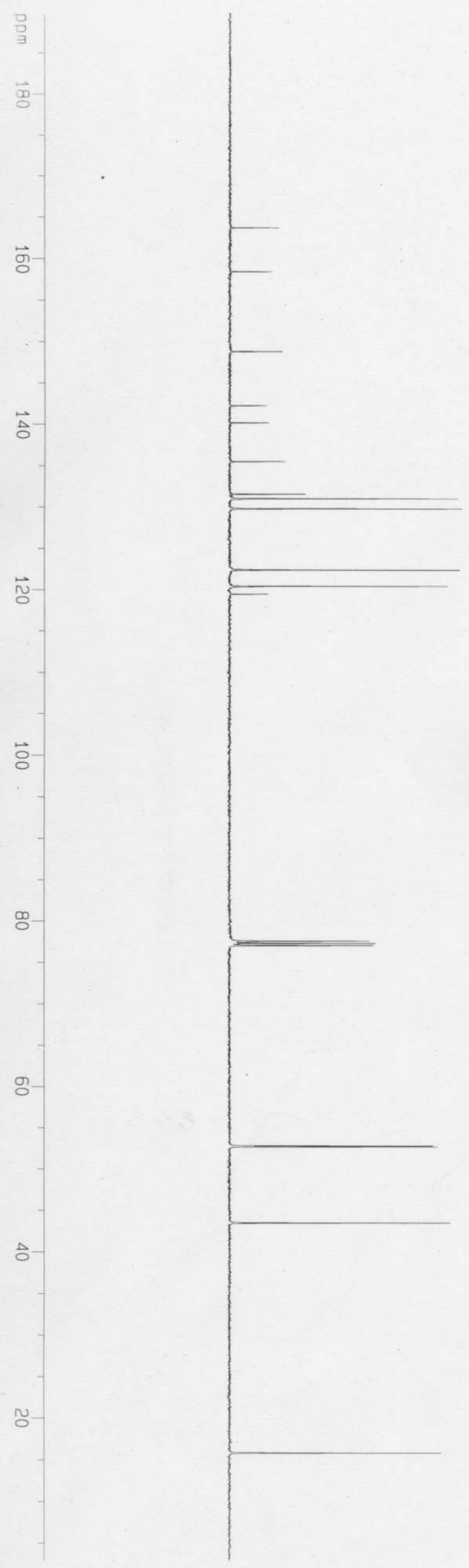

ppin

$-163.653$

$-158.372$

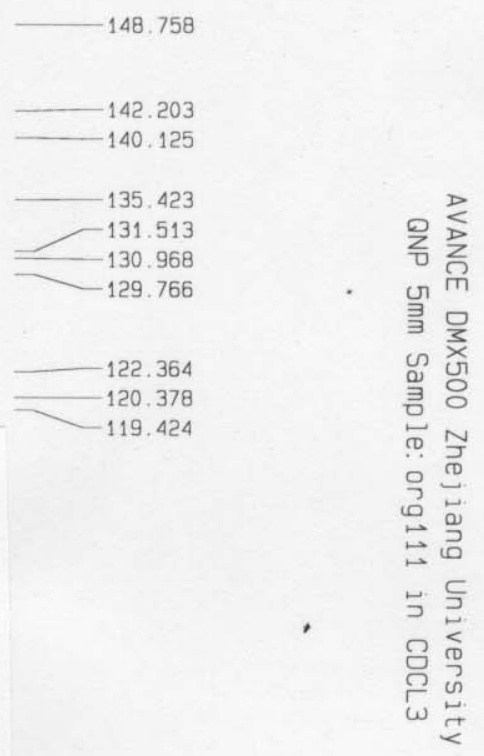<smiles>CCCc1oc(-c2cccc(C)c2)c(OC)c1OC</smiles>

52. 773

52. 690

43. 440

20 

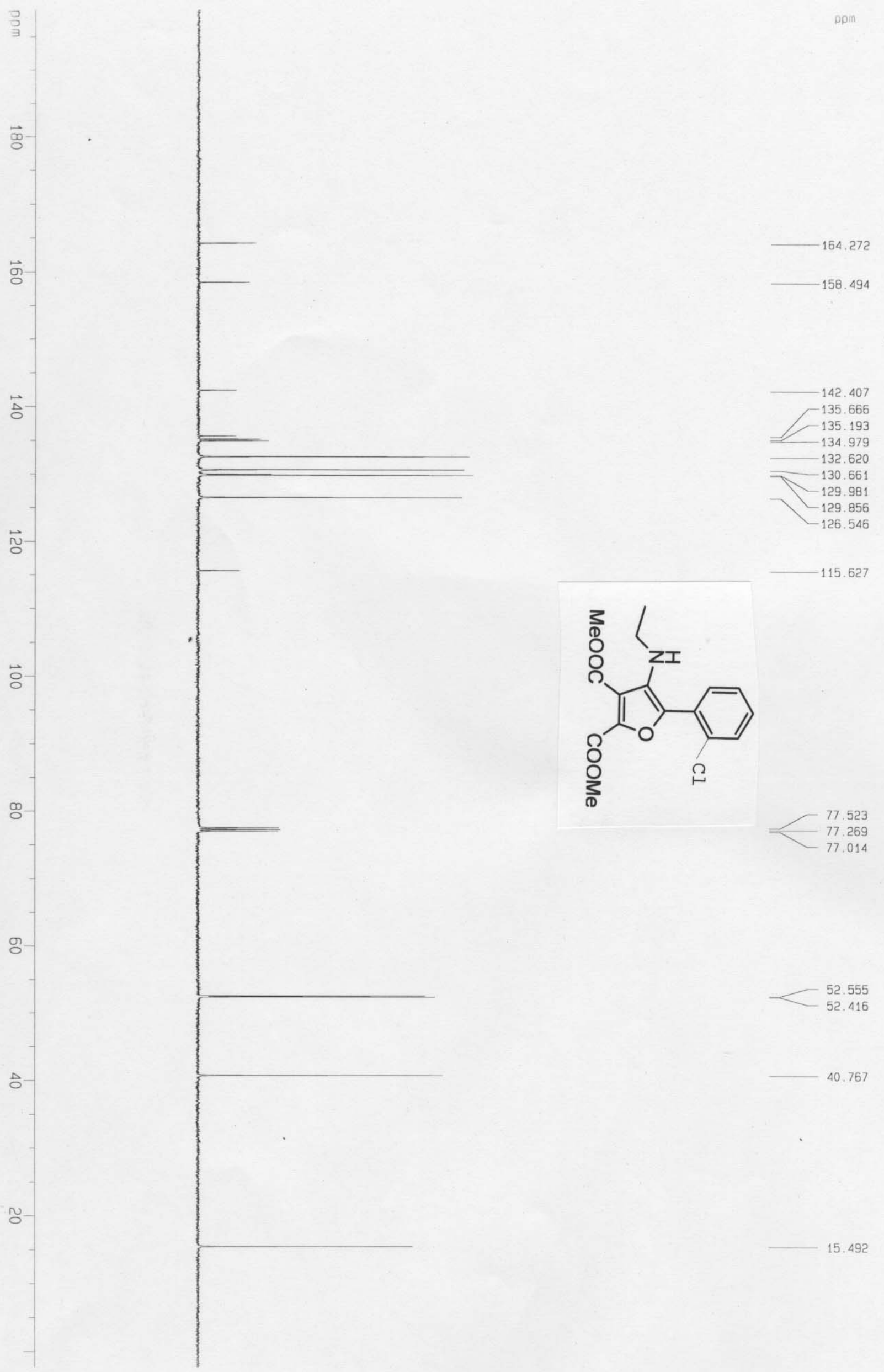


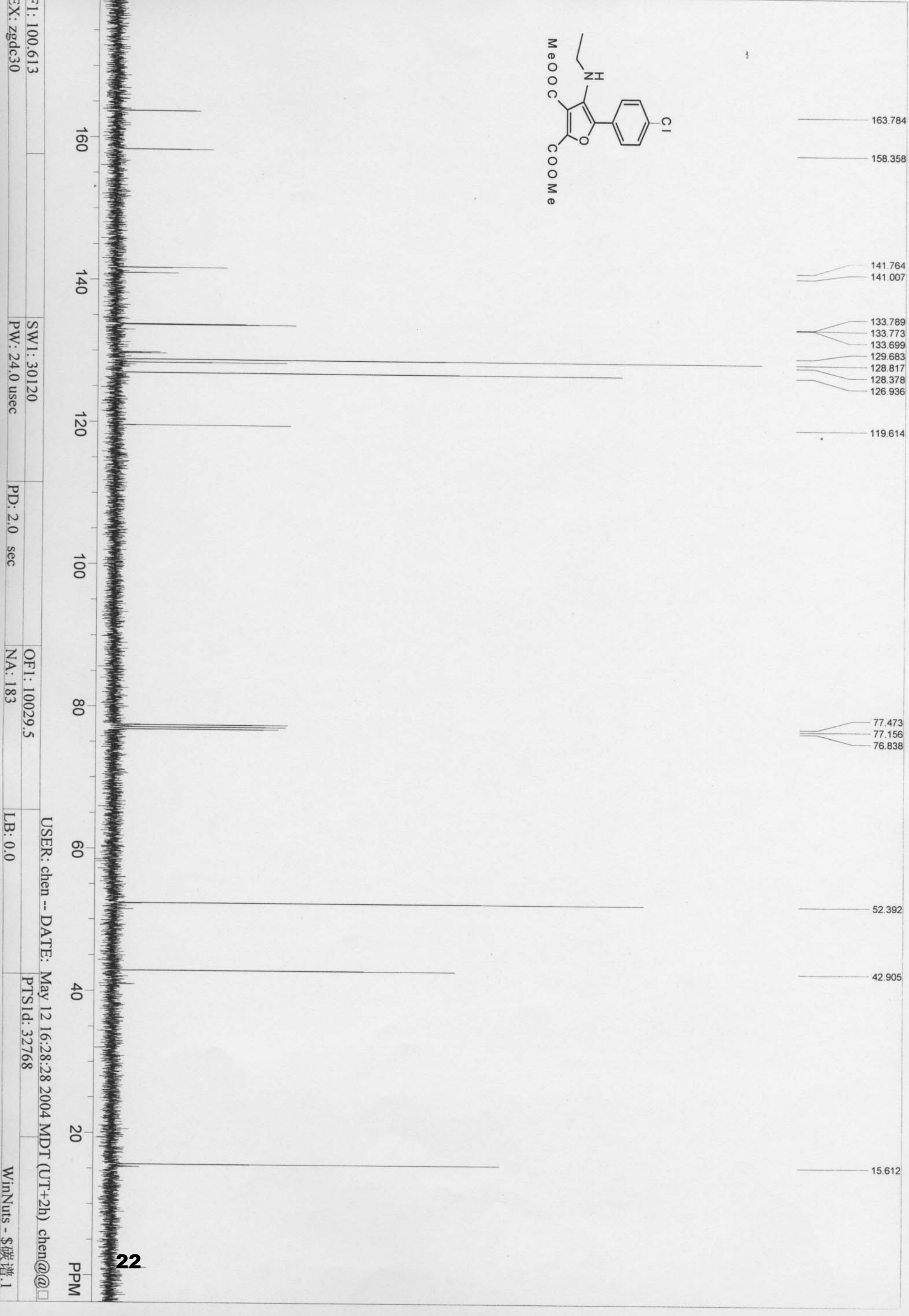




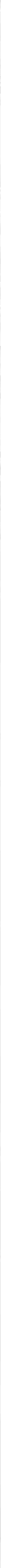



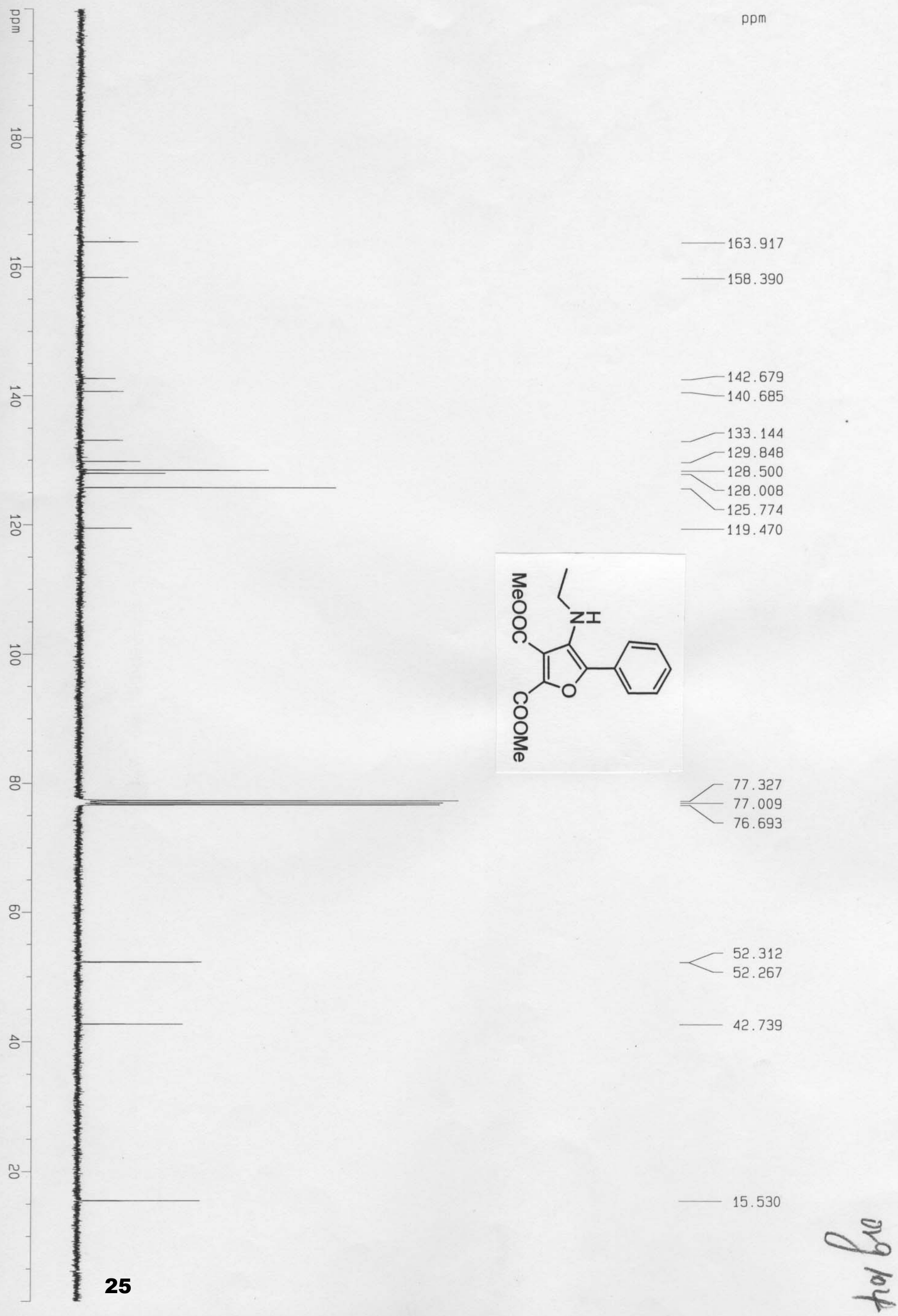


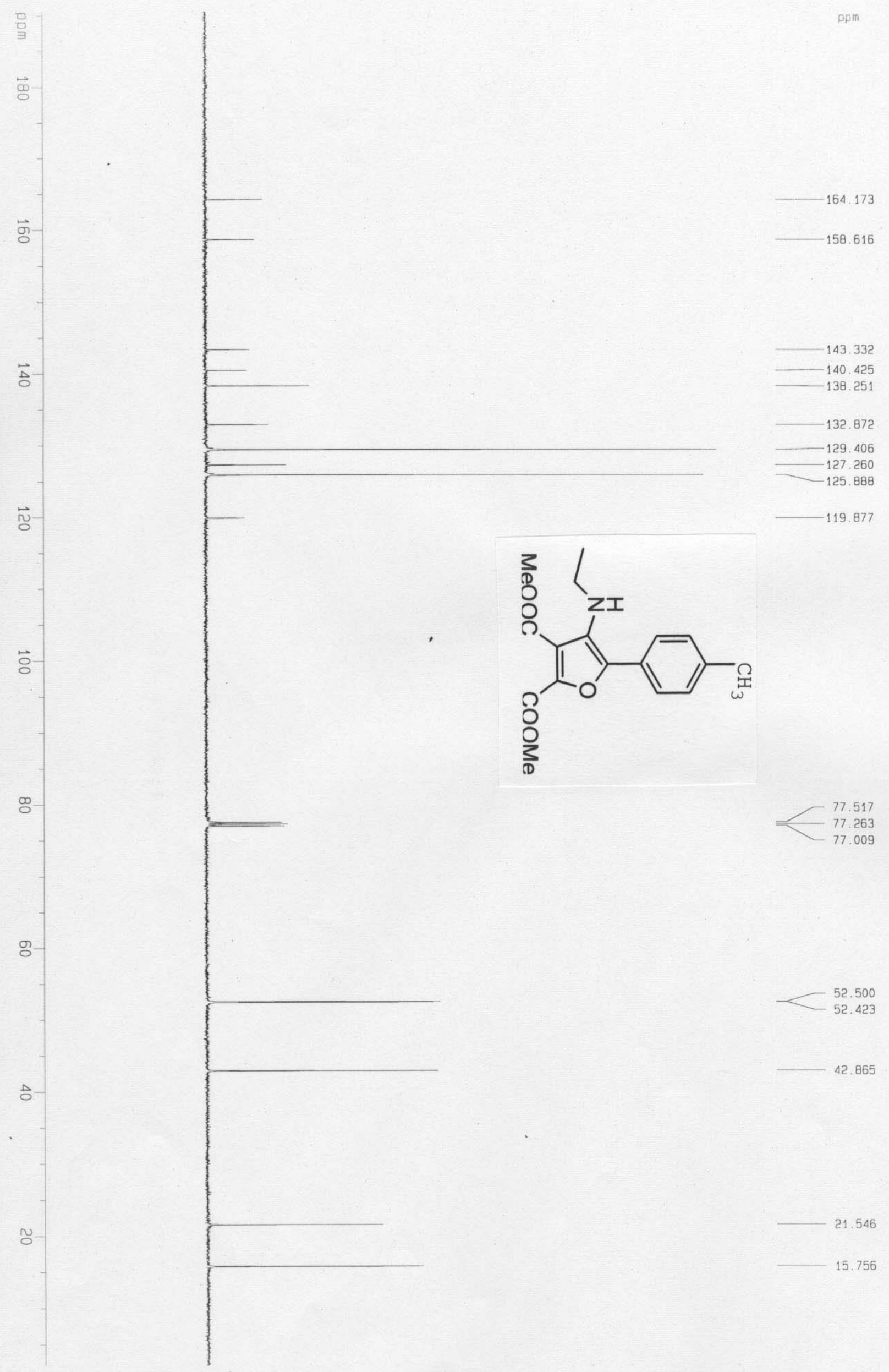




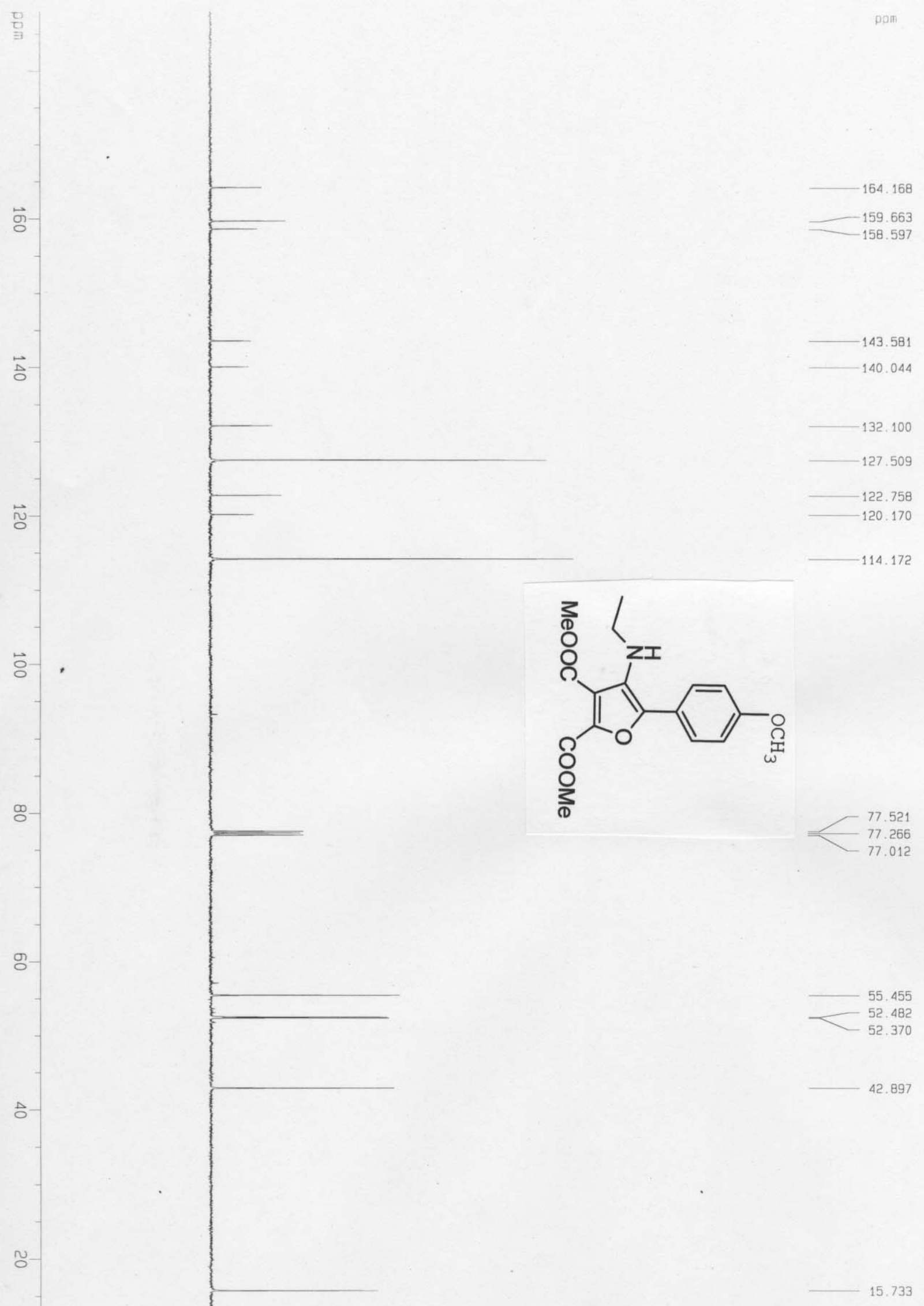



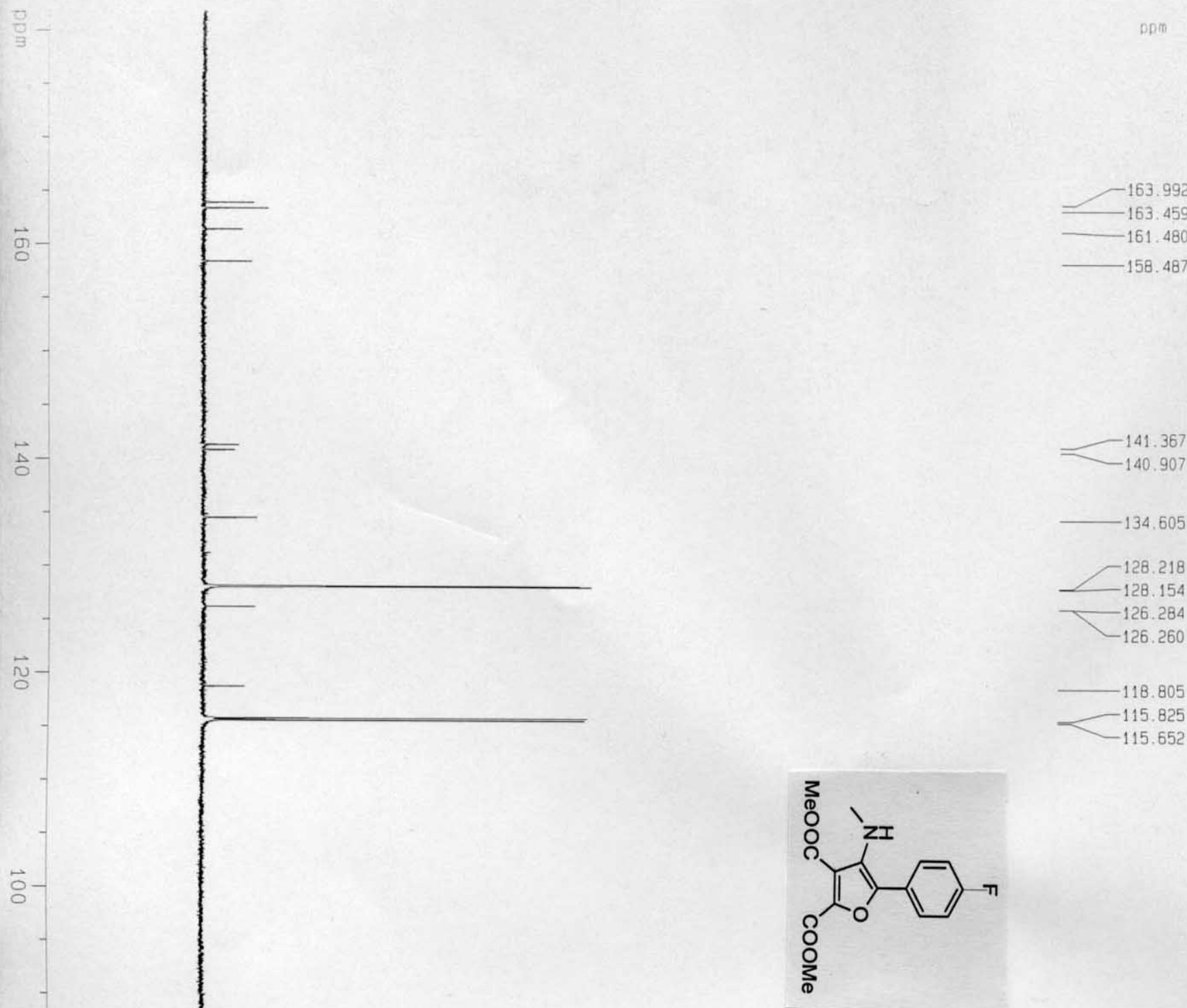

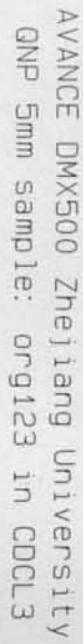

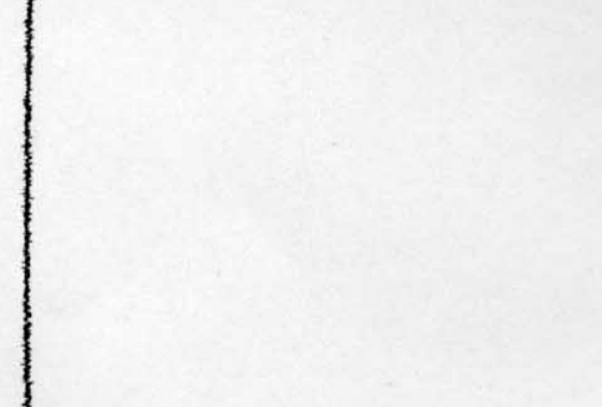




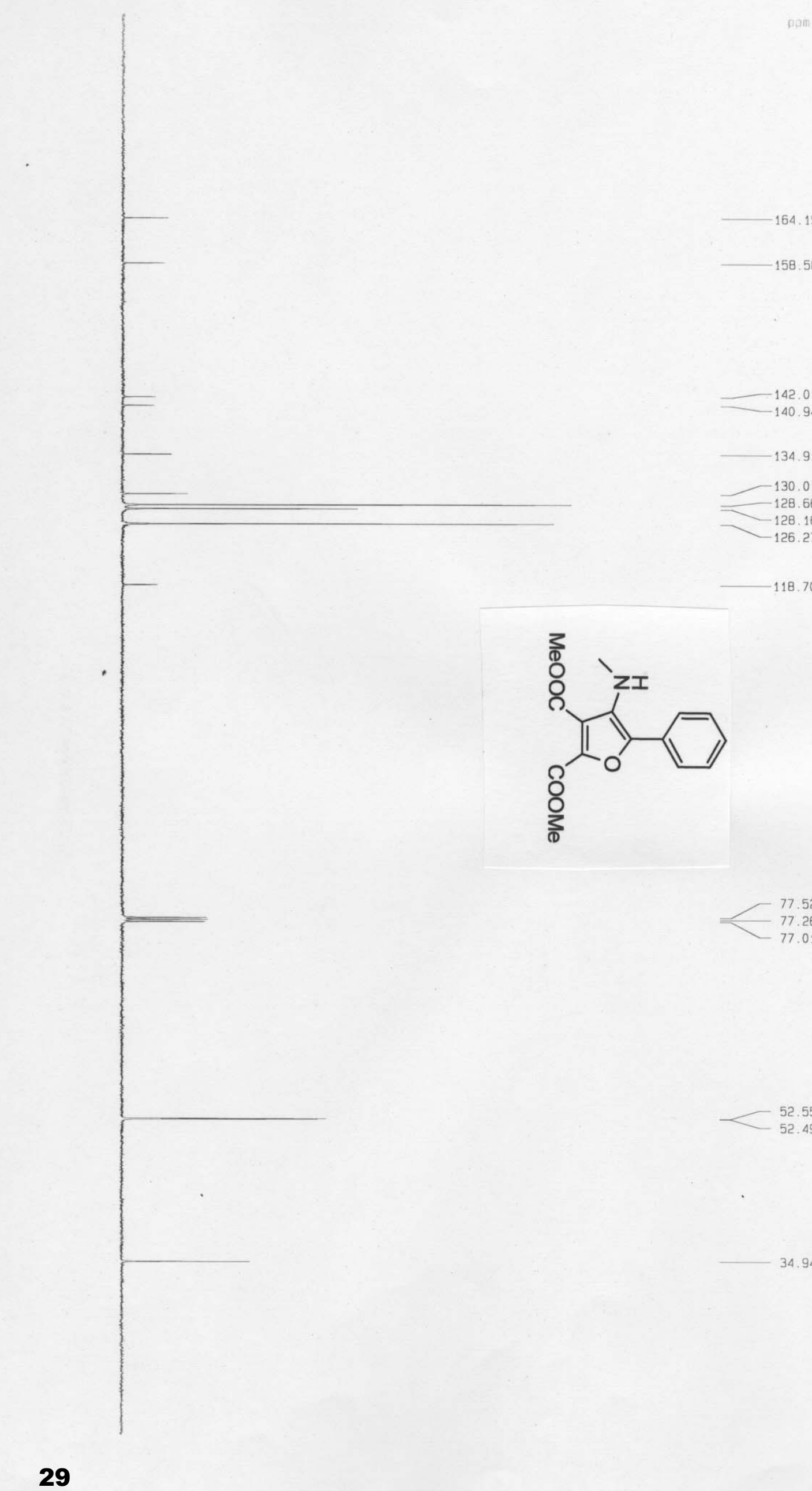



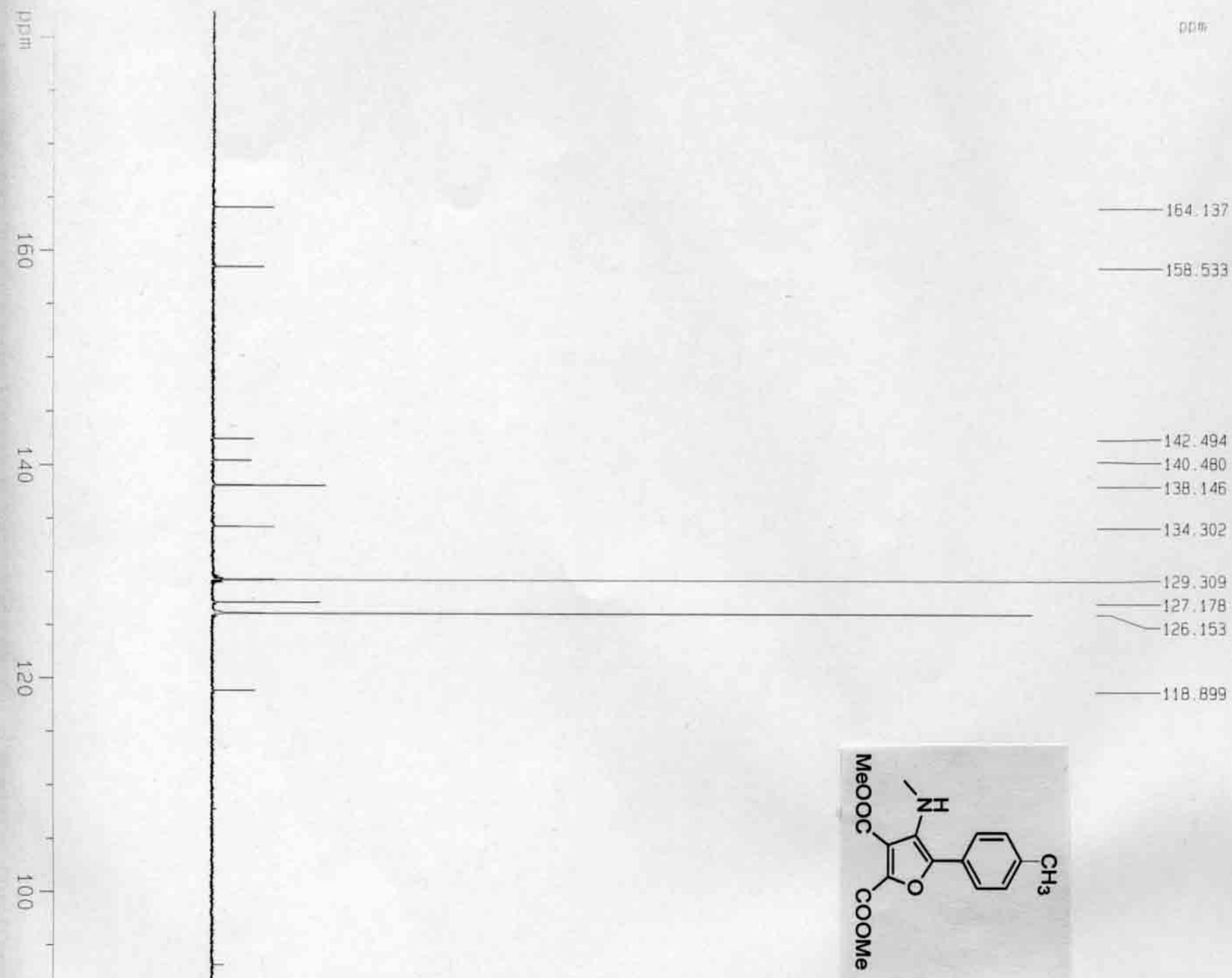

$$
\text { ( }
$$




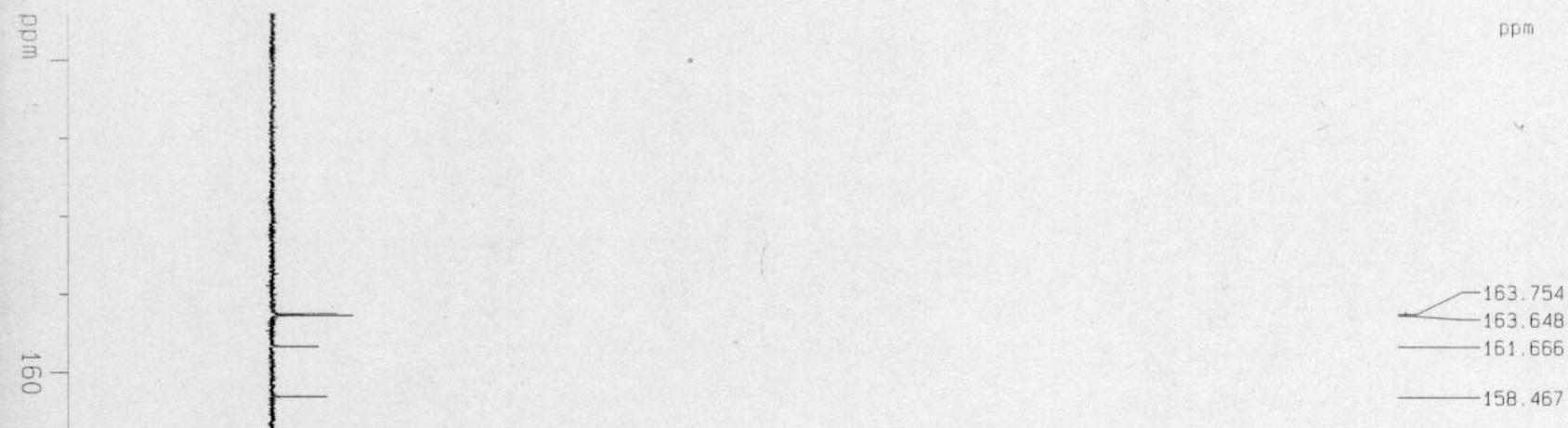

$-142.859$

$-140.756$ 139.137

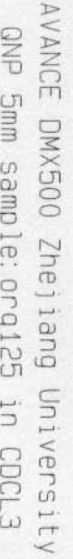
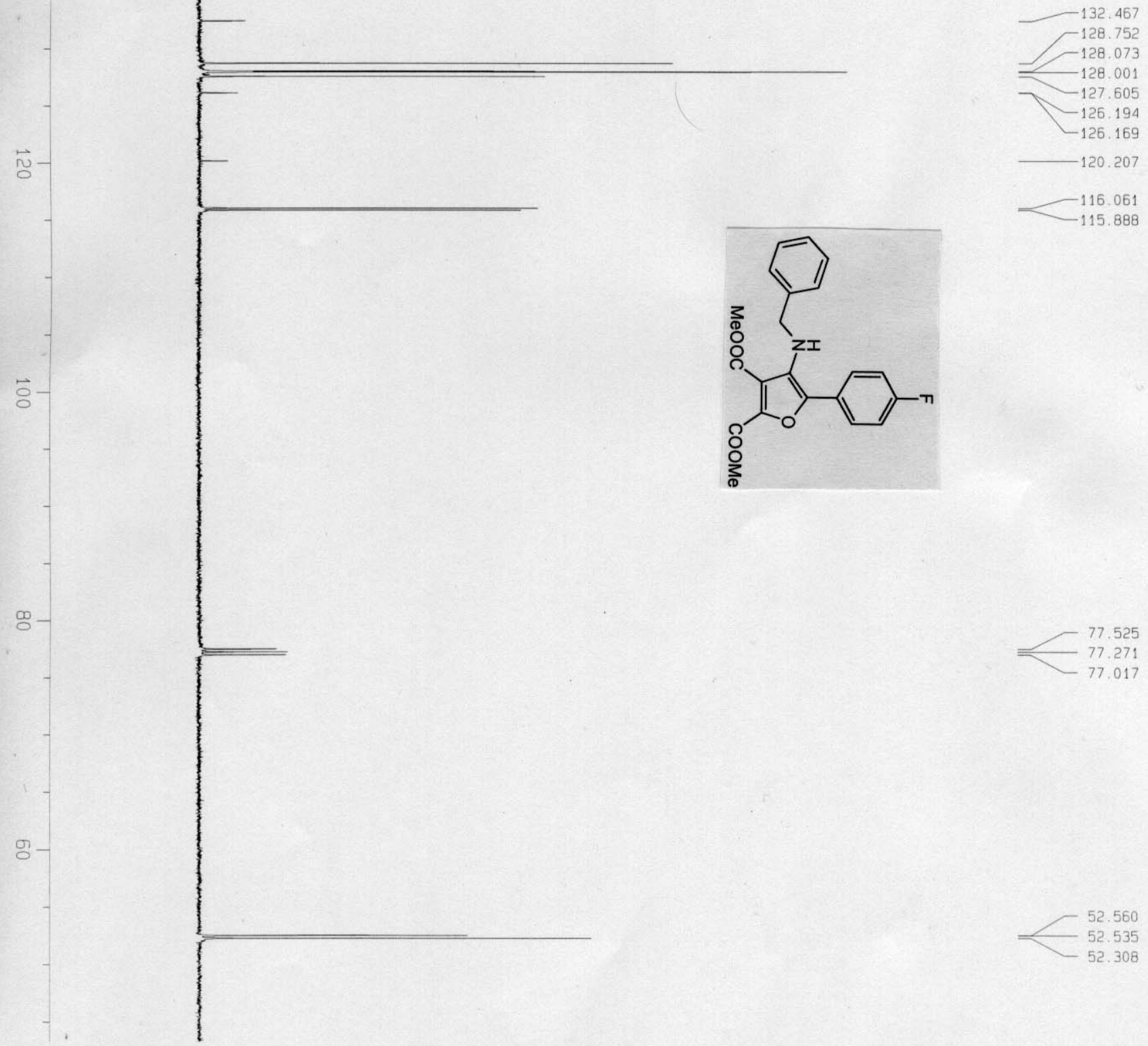

$-52.560$ 52.535 